\title{
Common gastrointestinal distress among infants: Role of optimal nutritional interventions
}

\author{
Dhanasekhar Kesavelu ${ }^{\mathrm{a}}$, Gaurav Sethi ${ }^{\mathrm{b}}$, Nikhil Bangale ${ }^{\mathrm{c}}$, Fahmina Anwar ${ }^{\mathrm{d}, *}$, \\ Shashidhar Rao ${ }^{\mathrm{d}}$ \\ a Apollo Children's Hospital, Chennai, Tamil Nadu, India \\ b Sethi Nursing Home, New Delhi, India \\ ${ }^{\mathrm{c}}$ Nestle Nutrition, Medical and Scientific Affairs, Mumbai, India \\ d Nestle Nutrition, Medical and Scientific Affairs, South Asia Region, Gurgaon, India
}

\section{A R T I C L E I N F O}

\section{Article history:}

Received 9 May 2017

Accepted 22 July 2017

Available online 24 July 2017

\section{Keywords:}

Breast milk

Gastrointestinal (GI) distress

Nutritional intervention

Probiotics

Regurgitation

\begin{abstract}
A B S T R A C T
The immature gastrointestinal (GI) tract makes infants vulnerable to transient disorders including colic, gastro-oesophageal reflux (GER) and constipation. These conditions cause a significant distress to infants and caregivers, and are among the most common reasons for the paediatrician referral. In addition, these conditions are associated with a significant morbidity, hospitalisations and high healthcare costs, adding to the burden of the disease. Although they are additions to the ongoing physiological development or arise from maladaptive behavioural responses, the evidence remains limited. The vague aetiology and the self-limiting nature, has devoid these conditions of robust clinical attention, both in the research and clinical practice. Frequent early-life intestinal insults may translate as a risk factor in the development of chronic GI disorders and psychological problems later in life. Emerging evidence suggests that providing an optimal nutritional intervention closer to the breast milk can be a path breaking approach to manage these frequently encountered conditions and aid the growth and development of infants.
\end{abstract}

(C) 2017 Published by Elsevier, a division of RELX India, Pvt. Ltd on behalf of INDIACLEN.

\section{Infantile GI distress: a synopsis}

Globally, prevalence of infantile colic is estimated to be approximately $10 \%-40 \%$ with a peak prevalence at 6 weeks. ${ }^{1}$ Approximately 70\%-85\% have regurgitation within the first two months of life, which generally resolves without intervention in $95 \%$ of the infants by one year. ${ }^{2,3}$ Constipation manifests in approximately $17 \%-40 \%$ of the infants during the first year of life, which gradually resolves in $60 \%$ of the infants after the age of 1 year. Despite the widespread prevalence, only $3 \%$ of the patients seek medical attention. ${ }^{4-6}$ The aetiology of colic, gastro-oesophageal reflux (GER) and constipation in infants remains largely unknown. ${ }^{1}$

Dysbiosis of the gut microflora, intolerance to cow's milk protein, lactose intolerance, Gastrointestinal (GI) immaturity, increased serotonin, improper feeding techniques, maternal smoking, delayed gastric emptying are some of the overarching

\footnotetext{
* Corresponding author.

E-mail addresses: drdskgastro@gmail.com (D. Kesavelu),

sethigaurav@hotmail.com (G. Sethi), Nikhil.Bangale@IN.nestle.com (N. Bangale), Fahmina.Anwar@in.nestle.com (F. Anwar), Shashidhar.Rao@IN.nestle.com (S. Rao).
}

factors identified to be associated with the GI distress among infants. ${ }^{1,7,8}$

\section{Gaps in the current treatment approaches}

The current management options for most of the GI distress disorders include a few standard approaches such as continued breastfeeding, counselling, reassurance and maternal dietary modification such as dietary exclusions. ${ }^{1,4,10}$ The ambiguity associated with the aetiology and pathogenesis of the infantile GI distress disorders limits the recommendations with most suggesting no intervention in the presence of positive weight gain and no associated red flags. $8,11,12$

In infants with colic, a change in formula or complementary intervention can be tried; however, soy formulas have been strongly discouraged. The probiotic strain Lactobacillus reuteri DSM 17938 has shown promising outcomes in the reduction of symptoms; however, requires an in depth research to strongly recommend its use. ${ }^{1,8,13}$ Modifications in the infants' diet such as decrease in the volume, thicker feeds and an increase in the frequency of feed has been identified to be beneficial in regurgitation. Switching to extensively hydrolysed protein or 
amino acid-based formula may be a suggested option. Antiregurgitation formulas specialised to improve uncomplicated regurgitation and quality of life can be used. ${ }^{10,14}$ The treatment of constipation is mainly focused on disimpaction of faeces and maintaining a regular bowel movement. Further diagnostics are required to distinguish between functional and organic constipations. ${ }^{4-6}$

The present gaps demand a need for concrete recommendations for the common GI distress among infants. ${ }^{8,11,12}$ Rome III diagnostics criteria for functional GI disorders used by Medical Professionals for the diagnosis of infantile GI distress, ${ }^{9}$ which has been discussed in Table 1.

\section{Limitations of traditionally used nutrition options}

In India, the complementary feeding practices are vastly suboptimal, as evidenced from the National Family Health Survey (NFHS) 3. Only 69\% of the infants younger than 2 months are exclusively breastfed, which declines to $28 \%$ between 4 and 5 months of age. The decline occurs because mothers supplement breast milk with plain water initially, and later with cow's milk. With the usage of cow's milk as the top replacement option for breast milk that is introduced as early as 4 months of age, it is clear that incorrect feeding practices are being followed in the country. ${ }^{15}$

Cow's milk can serve more harm than benefits. Cow's milk is a poor source of key nutrients such as iron, essential fatty acids, zinc, vitamin $C$, and niacin while has a higher saturated fatty acid content. Its low iron content as well as capacity to cause occult blood loss may increase the risk of anaemia. Further, the high amounts of calcium and casein can interfere with nutrient absorption. Despite the high amount, calcium absorption from cow's milk is estimated to be $20 \%$ lower than that of human milk. Moreover, the whey casein ratio in cow's milk is 20:80 compared to the optimal ratio of 60:40 in breast milk. Also, the lactose content in cow's milk is considerably lower than the breast milk, constituting approximately $50-70 \mathrm{~g} / \mathrm{L}$, thus a comparatively suboptimal energy source. The high protein and mineral content can lead to a high renal solute and urine concentration, which in the long term can cause dehydration. In addition, cow's milk protein allergy (CMPA), an immunological reaction to one or more milk proteins has been associated with long-term health consequences. ${ }^{16-21}$ Cow's milk can be a source of pathogenic bacteria such as Salmonella and Escherichia coli. ${ }^{22,23}$

\subsection{Need for quality nutrition closer to breast milk}

Breast milk is a gold standard offering an umbrella of benefits during infancy. Its specific nutritional characteristics and active support in nutritive, metabolic, immunological and protective functions makes it a vital component to help support GI health. ${ }^{14,18,19,24-27}$ (Table 2). Thus, in the unavailability of breast
Table 2

Properties of Breast Milk Specific to GI Health.

\begin{tabular}{ll}
\hline Nutritional characteristics & Content \\
\hline Whey Casein ratio & $60: 40$ \\
Calcium Phosphorus ratio & $2: 1$ \\
Osmolality & $300 \mathrm{mOsm} /$ \\
& $\mathrm{L}$ \\
Contains optimum vitamins, minerals, fats, lactose and probiotics & - \\
\hline
\end{tabular}

milk, offering nutritional interventions comparable to its composition can support digestive health. As conventional therapies have mainly focused on relief of symptoms, infantile GI distress requires an effective nutritional intervention over the pharmaceutical treatment. $^{28,29}$

\subsection{Suggested nutritional characteristics to alleviate infantile GI distress}

- Optimal lactose content

- Whey Casein ratio

- Calcium Phosphorus ratio

- Osmolality

- Probiotics + Prebiotics

\subsection{Optimal lactose content}

Lactose has an ability to attract water into the intestinal lumen, which produces an osmotic laxative effect. In addition, the prebiotic effect of lactose helps regulate intestinal microbiota. A study was conducted by Infante et al, to evaluate the impact of a formula with high levels of lactose in infants with functional constipation. Thirty healthy term-born, formula-fed infants, aged 4-10 weeks were included. Stool composition was measured by near-infrared reflectance analysis (NIRA). In addition, parents answered questions about crying associated with defecation and stool consistency at baseline and after two weeks of the adapted formula. The study resulted in a considerable reduction in the symptoms of constipation (Baseline $90 \%$ vs. after two weeks $10 \%$ ) such as hard stools and/or pain and difficulty during defection in infants (Fig. 1 ). ${ }^{30}$

In addition, studies have suggested that lactose-containing formulas show better absorption of key nutrients. Studies have suggested a $25 \%$ increase in copper absorption, $15 \%$ increase in calcium absorption $(P<0.01), 11 \%$ increase in manganese absorption $(P<0.05), 8 \%$ increase in magnesium absorption $(P<0.001)$ and $7 \%$ increase in zinc absorption, in the presence of lactose. ${ }^{31}$

Human milk is known to contain almost double the quantity of lactose compared with milk of other mammals. Breastfed infants have at least 8 points higher intelligence quotient in later life than non-breastfed ones. It also helps facilitating the synthesis of cerebrosides and myelination of the central nervous system. ${ }^{32}$ In

Table 1

Rome III criteria for functional GI disorders

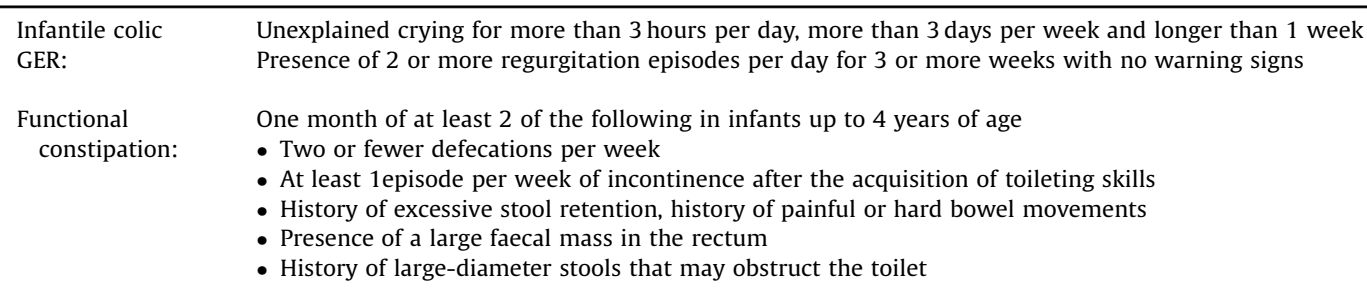




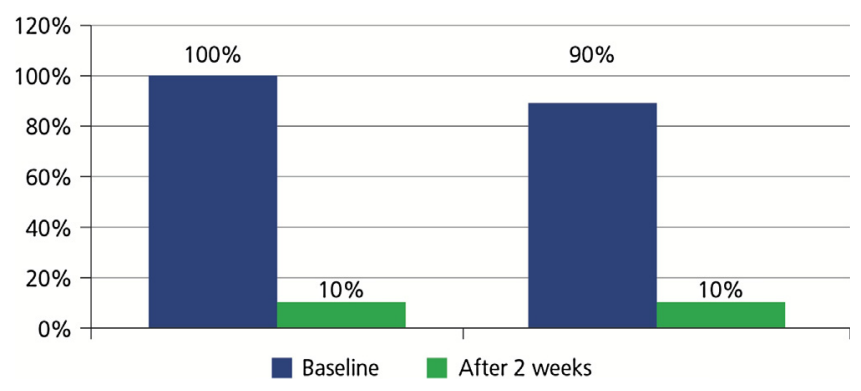

Fig. 1. Percentage of infants experiencing the symptoms of constipation before and after consumption of a formula, which contains lactose ${ }^{30}$.

Footnote: McNemar's test, p value: $<0.0001$, formula also contains magnesium. Source: Infante et al., Modification of stool's water content in constipated infants: management with an adapted infant formula, 2011.

addition, lactose undergoes fermentation forming lactic acid and short-chain fatty acids, which act as a substrate for the growth of the intestinal microbiota. ${ }^{33-35}$

\subsection{Whey Casein ratio}

Protein is known to have an impact in the process of gastric emptying, overriding the effects of osmolarity, fat and energy composition in a feed. Whey and casein, in an optimal ratio can help overcome delayed gastric emptying in infants. ${ }^{36-37}$ According to a study conducted by Billeaud et al. among 201 infant's $\leq 24$ months of age, gastric emptying differed significantly based on the type of feed. After $120 \mathrm{~min}$ of the feed, whey-based formula had a lower residual gastric activity (23\%) than casein-based formula (38\%) and cow's milk (44\%), second only to human milk (22\%) in infants with GER. ${ }^{38}$

In addition to evidence that the nature of protein in an infant's feed can affect the stool consistency, Malacaman et al. conducted a study feeding whey-predominate or casein-predominant formula over 16 weeks and evaluated soft stools based on parental observations. The percentage of hard stools was lesser among infants fed with whey formula (5\%) than with casein formula (16\%) at week $16 .^{39,40}$

Balmer et al. studied the faecal flora among infants receiving whey formula ( $n=33)$, casein formula $(n=29)$ and breastfed babies $(n=38)$ over 15 weeks. The study demonstrated that on day 14 , whey formula induced a faecal flora closer to that of breastfed babies compared with casein-predominant formula. ${ }^{41,42}$

\subsection{Calcium phosphorus ratio}

Evidence suggests that calcium: phosphorus ratio lowers than that of breast milk increases the risk of constipation and breast milk is ideal for gut comfort. Cow's milk has low calcium: phosphorus ratio of 1.2:1, a possible risk factor of hard stools. In addition, the formation of non-absorbable calcium palmitate soaps associated with cow's milk may also interfere with the bowel movement. $^{26,43,44}$

Khinchi et al. compared two different cow milk formulas with the same composition; however, with different calcium: phosphorous ratios (formula $A=2$ vs. formula $B=1.5$ ) over a period of 14 days among infants less than 6 months. It was noted that the mean number of hard stools for formula A was 0.9 (range 0-9) and for formula B it was 2.8 (range $0-15$ ). ${ }^{26}$

\subsection{Osmolality}

A higher osmolality may interfere with water absorption, the effect of which may range from diarrhoea to an increased risk of necrotising enterocolitis. ${ }^{45,46}$ The osmolality of the breast milk is approximately $300 \mathrm{mOsm} / \mathrm{L}$; hence, to remain closer to its osmolality, the American Academy of Pediatrics (AAP) recommends that the osmolality in formulas should be limited to $400 \mathrm{mOsm} / \mathrm{L}^{47}$

\subsection{Probiotics}

The positive treatment outcomes with probiotics and its associated safety profile make them a favourable alternative to current options in the management of GI distress. The ability of probiotics to support enteric flora through hits immunologic and metabolic functions contribute to the homoeostasis of the GI tract, regulate mucosal inflammation and gut motility and help immune function. Evidence suggests that providing probiotics or 'good bacteria' to infants shortly may reduce the development of GI disorders and have proven the efficacy in reducing the symptoms of colic in infants. Although more research is needed to confirm the findings before it can be recommended for infants, current evidence suggests that probiotics can confer maximum benefits with no adverse incidences. Among the common probiotic strains, $L$. reuteri has shown promising results in the treatment of GI distress disorders through its ability to help immune cells contribute its tolerance in the gut. It reduces the production of pro-inflammatory cytokines macrophages, monocytes and dendritic cells. Furthermore, it promotes the development of regulatory $\mathrm{T}$ cells producing interleukin-10 and tumour growth factor-beta. ${ }^{11,12,48}$

A study was conducted by Savino et al. to test the hypothesis that oral administration of Lactobacillus reuteri would improve symptoms of infantile colic. Ninety colicky infants were randomly assigned to receive $L$. reuteri (American Type Culture Collection strain 55730) at a dose of 108 colony-forming units in 5 drops, $30 \mathrm{~min}$ after feeding, once per day or simethicone at a dose of $60 \mathrm{mg} /$ day in 15 drops twice per day, after feeding, for 28 days. The results showed a reduction in the crying time of infants supplemented with $L$. reuteri ( $51 \mathrm{~min} /$ day) when compared to simethicone ( $145 \mathrm{~min} /$ day). No adverse effects were reported (Fig. 2). ${ }^{49}$

According to a study conducted by Indrio et al. among 589 infants, prophylactic use of probiotic strain reuteri DSM 17938 reduced symptoms of infantile GI distress including colic, regurgitation and constipation (Fig. 3$).^{50}$

\subsection{Prebiotics}

Prebiotics are known to increase microbial mass, induce selective fermentation and production of short chain fatty acids

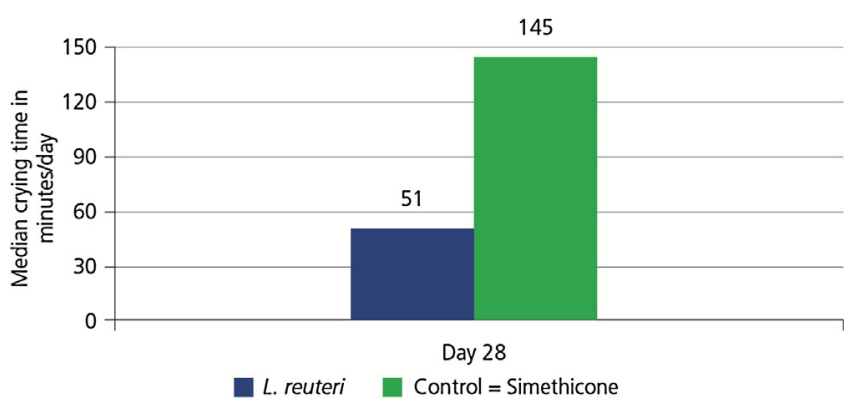

Fig. 2. Crying time of infants supplemented with $L$. reuteri and simethicone. Source: Savino et al. ${ }^{49}$ Lactobacillus reuteri (American Type Culture Collection Strain 55730) Versus Simethicone in the Treatment of Infantile Colic: A Prospective Randomized Study. 

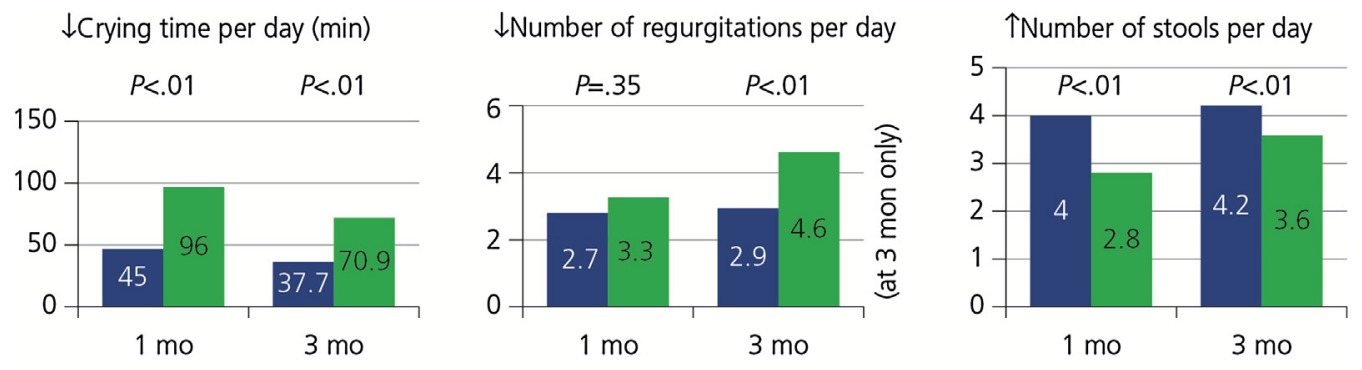

L. reuteri $(n=238) \quad$ Placebo $(n=230)$

Fig. 3. Probiotics in the prevention of infantile GI distress.

Source: Indrio et al. ${ }^{50}$ Prophylactic use of a probiotic in the prevention of colic, regurgitation, and functional constipation.

(SCFA), stimulate GI motility, mucosal blood flow and oxygen uptake of the intestinal mucosa. ${ }^{51,52}$

Vivatvakin et al. conducted a study in 2010 among 144 healthy, full-term infants to compare whey formula containing long-chain polyunsaturated fatty acids, galacto-oligosaccharides and fructooligosaccharides (experimental formula) and a casein formula without additional ingredients as feeding options (control). Infants were fed with formulas for 30 days. The results suggested that prebiotic-containing whey-based formula provides superior GI comfort than a control formula (Fig. 4). ${ }^{52}$

\subsection{Clinical relevance}

Current understanding of infantile GI distress in infants clearly suggests that these conditions have myriad aetiology and pathogenesis, with no general consensus available. The clinical focus towards managing infantile GI distress among infants' remains limited due to their presumed self-limiting nature. Most recommendations suggest that therapeutic management should be reserved for GI discomfort with complications. However, the associated psychological stress often leads to a never-ending cycle of trying medications, dietary manipulations, behavioral strategies and complementary supplements among parents, caregivers as well as health care providers.

Emerging evidence suggests that nutritional management could be a feasible option for both prevention as well as management of distressful GI symptoms. The primary nutritional considerations should include providing a solution closer to the properties of breast milk, the nutrient nectar for the infant. As it is clear that cow's milk does not have the right feed characteristics, it is vital to utilize a nutritional solution closer to breast milk. Thus focusing on the proposed elements of the feeding characteristics such as whey casein ratio, calcium phosphorus ratio, osmolality, lactose content and probiotics in optimal and regulated amounts can confer an umbrella of benefits in alleviating the symptoms of GI distress. Moreover, emerging evidences are in favor of the benefits conferred with the use of probiotics, with no adverse reports. Although the current evidence remains limited, further
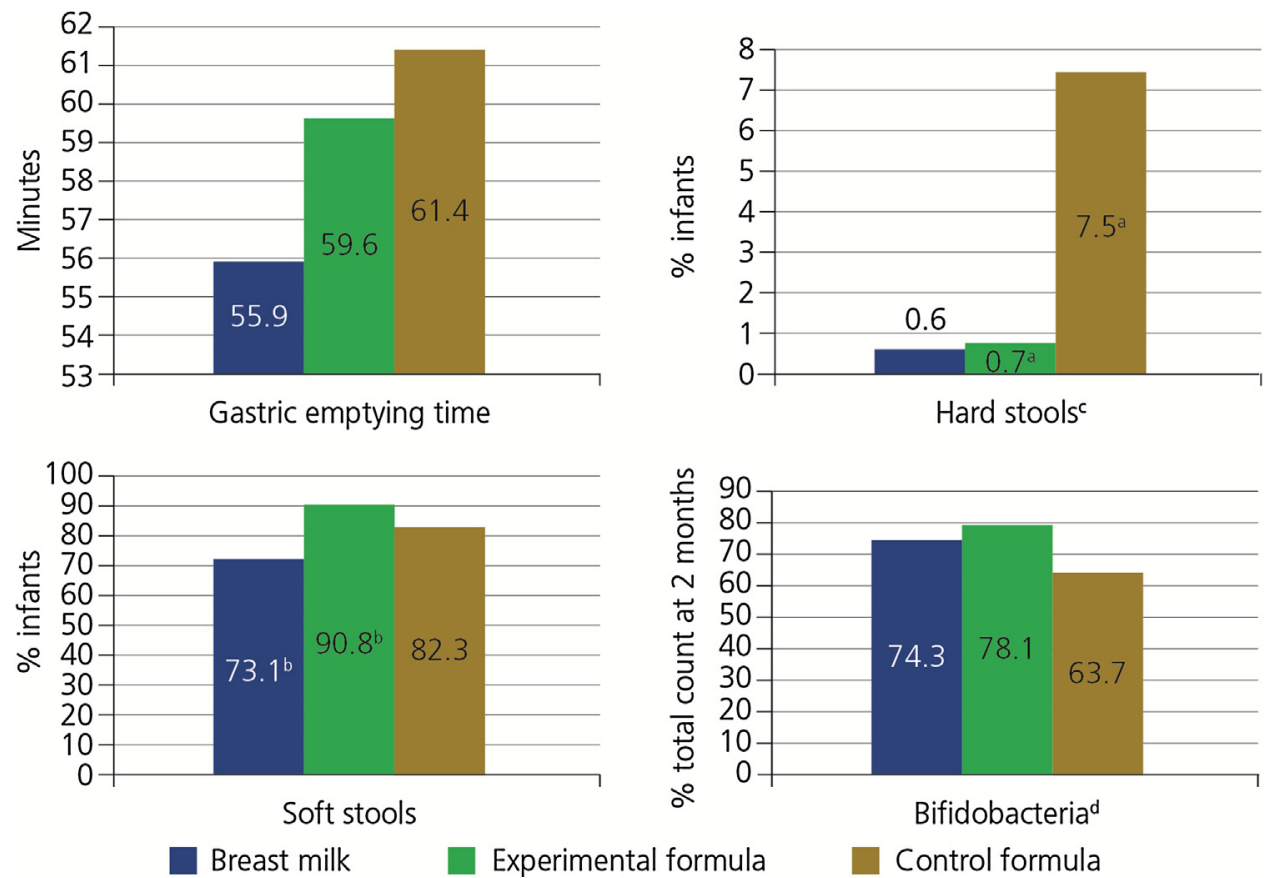

Fig. 4. Use of prebiotics to optimise the GI health in infants.

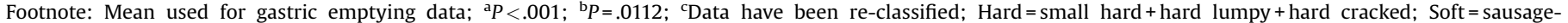
like + soft + loose; ${ }^{d}$ Bacterial count $=\log$ colony-forming units/g as determined by fluorescence in-situ hybridisation.

Source: Vivatvakin ${ }^{52}$ Effect of a whey-predominant starter formula containing LCPUFAs and oligosaccharides (FOS/GOS) on GI comfort in infants. 
research is required to reinforce the efficacy and to formulate definitive recommendations.

\section{Conflict of interest}

The authors have none to declare.

\section{Acknowledgement}

We thank the members of medical and scientific affair, Nestle South Asia team for support and assistance in development of the manuscript.

\section{References}

1. Johnson JD, Cocker K, Chang E. Infantile colic: recognition and treatment. Am Fam Phys. 2015;92(October (7)).

2. Czinn SJ, Blanchard S. Gastroesophageal reflux disease in neonates and infants. Pediatr Drugs. 2013;15(February (1)):19-27.

3. Koda YK, Ozaki MJ, Murasca K, Vidolin E. Clinical features and prevalence of gastroesophageal reflux disease in infants attending a pediatric gastroenterology reference service. Arquivos de gastroenterologia. 2010;47 (March (1)):66-71.

4. Baker SS, Liptak GS, Coletti RB. Constipation guideline committee of the North American Society for Pediatric Gastroenterology, Hepatology and Nutrition Evaluation and treatment of constipation in infants and children: recommendations of the North American Society for Pediatric Gastroenterology, Hepatology and Nutrition. J Pediatr Gastroenterol Nutr. 2006;43(3):e1-3.

5. Pijpers MA, Bongers ME, Benninga MA, Berger MY. Functional constipation in children: a systematic review on prognosis and predictive factors. J Pediatr Gastroenterol Nutr. 2010;50(March (3)):256-268.

6. Biggs WS, Dery WH. Evaluation and treatment of constipation in infants and children. Am Fam Phys. 2006;73(February (3)):469-477.

7. Laubereau B, Filipiak-Pittroff B, Von Berg A, et al. Caesarean section and gastrointestinal symptoms, atopic dermatitis, and sensitisation during the first year of life. Arch Dis Child. 2004;89(November (11)):993-997.

8. Cohen GM, Albertini LW. Colic. Pediatr Rev/Am Acad Pediatr. 2012;33(7):332.

9. ROME III Diagnostic Criteria for Functional Gastrointestinal Disorders. Rome criteria. http://www.romecriteria.org/assets/pdf/19 Romell apA 885-898.pdf.

10. Baird DC, Harker DJ, Karmes AS. Diagnosis and treatment of gastroesophageal reflux in infants and children. Am Fam Phys. 2015;92(October (8)):705-714.

11. Whitfield KL, Shulman RJ. Treatment options for functional gastrointestina disorders from empiric to complementary approaches. Pediatr Ann. 2009;38 (May (5)).

12. Savino F, Tarasco V. New treatments for infant colic. Curr Opin Pediatr. 2010;22 (December (6)):791-797.

13. Roberts D, Ostapchuk M, O'brien J. Infant Colic. 2004;70:735-742.

14. Vandenplas Y, Rudolph CD, Di Lorenzo C, et al. Pediatric gastroesophageal reflux clinical practice guidelines: joint recommendations of the North American Society for Pediatric Gastroenterology, Hepatology, and Nutrition (NASPGHAN) and the European Society for Pediatric Gastroenterology, Hepatology, and Nutrition (ESPGHAN). J Pediatr Gastroenterol Nutr. 2009;49 (October (4)):498-547.

15. Arnold F, Parasuraman S, Arokiasamy P, Kothari M. National Family Health Survey (NFHS-3) India 2005-06. Mumbai: International Institute for Population Sciences, Ministry of Health and Family Welfare, Government of India; 2009.

16. EFSA N. Panel. Scientific opinion on the essential composition of infant and follow-on formulae. EFSA J. 2014;12:3760.

17. American Society for Clinical Nutrition. Lactose content of milk and milk products. Am J Clin Nutr. 1988;48:1099-1104 Chapter 3.

18. Dionex Measuring Lactose in Milk: A Validated Method. http://www.dionex. com/en-us/webdocs/110944-AU182-HPLC-Lactose-Milk-29Jul2011-LPN2894. pdf.

19. Ziegler EE. Adverse effects of cow's milk in infants. Issues in Complementary Feeding, vol. 60Karger Publishers; 2007:185-199.

20. Ivakhnenko O, Nyankovskyy S. Nutritional status of babies and influence of unmodified cow's milk on allergic reactions according to the epidemiological study from Ukraine. Pediatr Polska. 2013;88(April (2)):138-143.

21. Lien EL. Infant formulas with increased concentrations of $\alpha$-lactalbumin. Am J Clin Nutr. 2003;77(June (6)):1555S-1558S.

22. Microbiological risk assessment of raw cow milk Food Standards. https://www. foodstandards.gov.au/code/proposals/documents/P1007\%20PPPS\%20for\% 20raw\%20milk\%201AR\%20SD1\%20Cow\%20milk\%20Risk\%20Assessment.pdf.
23. Kumar AM, Murugalatha N. Isolation of Lactobacillus plantarum from cow milk and screening for the presence of sugar alcohol producing gene. $J$ Microbiol Antimicrob. 2012;4(January (1)):16-22.

24. Leung AK, Sauve RS. Breast is best for babies. J Natl Med Assoc. 2005;97(July (7)): 1010 .

25. Motee A, Jeewon R. Importance of exclusive breastfeeding and complementary feeding among infants. Curr Res Nutr Food Sci. 2014;2(August (2)):56.

26. Khinchi MS, Deurell M, Hejl M, Hjelt K. Ca:P ratio in cows milk formula. The influence on regurgitation, vomiting, bowel habits and uneasiness in healthy newborns less than 6 months old. J Pediatr Gastroenterol Nutr. 1999;28(May (5)):591.

27. Pearson F, Johnson MJ, Leaf AA. Milk osmolality: does it matter? Arch Dis ChildFetal Neonatal Ed. 2013;98(March (2)):F166-F169.

28. Guaraldi F, Salvatori G. Effect of breast and formula feeding on gut microbiot a shaping in newborns. Human gut microbiota: onset and shaping through life stages and perturbations. :11.

29. Donovan S, Gibson G, Newburg D. Prebiotics in infant nutrition. Mead Johson \& Company; 2009:1-42.

30. Infante DD, Segarra OO, Redecillas SS, Alvarez MM, Miserachs MM. Modification of stool's water content in constipated infants: management with an adapted infant formula. Nutr J. 2011;10(May(1)):55.

31. Abrams SA, Griffin IJ, Davila PM. Calcium and zinc absorption from lactosecontaining and lactose-free infant formulas. Am J Clin Nutr. 2002;76(August (2)):442-446.

32. Singh M. Nutrition, brain and environment: how to have smarter babies? Indian Pediatr. 2003;40(3):213-220.

33. Vonk RJ, Reckman GA, Harmsen HJ, Priebe MG. Probiotics and lactose intolerance. INTECH Open Access Publisher; 2012.

34. Adolfsson O, Meydani SN, Russell RM. Yogurt and gut function. Am J Clin Nutr. 2004;80(August (2)):245-256.

35. Szilagyi A. Lactose-a potential prebiotic. Ali Pharmacol Ther. 2002;16:15911602.

36. Lönnerdal B. Nutritional and physiologic significance of human milk proteins. Am J Clin Nutr. 2003;77(June (6)):1537S-1543S.

37. Khoshoo V, Brown S. Gastric emptying of two whey-based formulas of different energy density and its clinical implication in children with volume intolerance. Eur J Clin Nutr. 2002;56(July (7)):656.

38. Billeaud C, Guillet J, Sandler B. Gastric emptying in infants with or without gastro-oesophageal reflux according to the type of milk. Eur J Clin Nutr. 1990;44(August (8)):577-583.

39. Bae SH. Diets for constipation. Pediatr Gastroenterol Hepatol Nutr. 2014;17 (December (4)):203-208.

40. Malacaman EE, Abbousy FK, Crooke D, Nauyok Jr. GJr.. Effect of protein source and iron content of infant formula on stool characteristics. J Pediatr Gastroenterol Nutr. 1985;4(October (5)):771-773.

41. Balmer SE, Scott PH, Wharton BA. Diet and faecal flora in the newborn: casein and whey proteins. Arch Dis Child. 1989;64(December (12)):1678-1684.

42. Rochat F, Cherbut C, Barclay D, et al. A whey-predominant formula induces fecal microbiota similar to that found in breast-fed infants. Nutr Res. 2007;27 (December (12)):735-740.

43. Greer FR. Calcium, phosphorus, and magnesium: how much is too much for infant formulas? J Nutr. 1989;119(December (2 Suppl.)):1846-1851.

44. Chapter11. Calcium.Fao.org 2016. http://www.fao.org/docrep/004/y2809e/ y2809e0h.htm (Published 29 March 2016).

45. Field M. Intestinal ion transport and the pathophysiology of diarrhea. J Clin Investig. 2003;111(April (7)):931-943.

46. Woods TA. Diarrhea. In: Walker HK, Hall WD, Hurst JW, eds. Clinical methods: the history, physical, and laboratory examinations. 3rd ed. Boston: Butterworths; 1990. Chapter 88, Available from: https://www.ncbi.nlm.nih.gov/books/ NBK414/.

47. Jew RK, Owen D, Kaufman D, Balmer D. Osmolality of commonly used medications and formulas in the neonatal intensive care unit. Nutr Clin Pract. 1997; 12(August (4)):158-163.

48. Anabrees J, Indrio F, Paes B, AlFaleh K. Probiotics for infantile colic: a systematic review. BMC Pediatr. 2013;13(November (1)):186.

49. Savino F, Pelle E, Palumeri E, Oggero R, Miniero R. Lactobacillus reuteri (American Type Culture Collection Strain 55730) versus simethicone in the treatment of infantile colic: a prospective randomized study. Pediatrics. 2007;119(January (1)):e124-30.

50. Indrio F, Di Mauro A, Riezzo G, et al. Prophylactic use of a probiotic in the prevention of colic, regurgitation, and functional constipation: a randomized clinical trial. JAMA Pediatr. 2014;168(March (3)):228-233.

51. Scholtens PA, Goossens DA, Staiano A. Stool characteristics of infants receiving short-chain galacto-oligosaccharides and long-chain fructo-oligosaccharides: a review. World J Gastroenterol: WJG. 2014;20(October (37)):13446.

52. Vivatvakin B, Mahayosnond A, Theamboonlers A, Steenhout PG, Conus NJ. Effect of a whey-predominant starter formula containing LCPUFAs and oligosaccharides (FOS/GOS) on gastrointestinal comfort in infants. Asia Pac J Clin Nutr. 2010;19(December (4)):473-480. 